\title{
Investigating the true nature of three hard X-ray sources
}

\author{
M. Molina ${ }^{1 \star}$ A. Malizia ${ }^{1}$ N. Masetti ${ }^{1,3}$ L. Bassani ${ }^{1}$ A. Bazzano ${ }^{2}$ A.J. Bird ${ }^{4}$ M. Fiocchi ${ }^{2}$ E. Palazzi ${ }^{1}$ \\ P. Ubertini ${ }^{2}$ \\ ${ }^{1}$ OAS/INAF, ia Gobetti 101, I-40129 Bologna, Italy \\ ${ }^{2}$ IAPS/INAF, via Fosso del Cavaliere 100, I-00133 Rome, Italy \\ ${ }^{3}$ Departamento de Ciencias Físicas, Universidad Andrés Bello, Fernández Concha 700, Las Condes, Santiago, Chile \\ ${ }^{4}$ Department of Physics and Astronomy, University of Southampton, Highfield, Southampton SO17 1BJ, UK
}

Accepted XXX. Received YYY; in original form ZZZ

\begin{abstract}
Many of the new high energy sources discovered both by INTEGRAL/IBIS and Swift/BAT have been characterised thanks to extensive, multi-band follow-up campaigns, but there are still objects whose nature remains to be asserted. In this paper we investigate the true nature of three high energy sources, IGR J12134-6015, IGR J16058-7253 and Swift J2037.2+4151, employing multiwavelength data from the NIR to the X-rays. Through Gaia and ESO-VLT measurements and through Swift/XRT X-ray spectral analysis, we re-evaluate the classification for IGR J12134-6015, arguing that the source is a Galactic object and in particular a Cataclysmic Variable. We were able to confirm, thanks to NuSTAR observations, that the hard X-ray emission detected by INTEGRAL/IBIS and Swift/BAT from IGR J16058-7253 is coming from two Seyfert 2 galaxies which are both counterparts for this source. Through optical and X-ray spectral analysis of Swift J2037.2+4151 we find that this source is likely part of the rare and peculiar class of Symbiotic X-ray binaries and displays flux and spectral variability as well as interesting spectral features, such as a blending of several emission lines around the iron line complex.
\end{abstract}

Key words: Methods: data analysis - X-rays: binaries - galaxies: Seyfert

\section{INTRODUCTION}

In the past 20 years, our knowledge of the high energy sky has greatly improved thanks to missions such as INTEGRAL (Winkler et al. 2003) and the Neil Gehrels Swift Observatory (Gehrels et al. 2004), that with their two main detectors, IBIS (Ubertini et al. 2003) and BAT (Barthelmy et al. 2005), have been continuously monitoring the sky above $14 \mathrm{keV}$. Both missions have produced several catalogues, populated by both known sources and objects that are completely new high energy emitters and whose nature is yet to be determined. Many of these new hard X-ray sources are common between the INTEGRAL/IBIS (e.g. Bird et al. 2006, 2007, 2010, 2016) and the Swift/BAT catalogues (e.g. Baumgartner et al. 2010, 2013; Oh et al. 2018). In particular in all the INTEGRAL/IBIS surveys released so far (Bird et al. 2016 and Krivonos et al. 2021 and references therein), about $20 \%$ of the detected high energy sources were completely new discoveries with no clear counterpart and no firm classification.

In the past years, many of these sources have been characterised through an extensive follow-up campaign, both in the optical and in the X-ray band resulting in many papers published since 2004 (see e.g. Masetti et al. 2013 and Landi et al. 2017). The variety of sources thus uncovered is truly remarkable, spanning from X-ray binaries like Low Mass X-ray Binaries (LMXB, see Sazonov et al. 2020 for a review) and High Mass X-ray Binaries (HMXB, see Kretschmar et al. 2019 for a review), Cataclysmic Variables (CVs, see Lutovinov

^ E-email: manuela.molina@inaf.it (MM) et al. 2020 for a review) and Symbiotic X-ray Binaries (e.g. Masetti et al. 2007) to extragalactic objects, mainly Active Galactic Nuclei (AGN; see Malizia et al. 2020 for a review).

Follow-up campaigns of new IBIS and BAT sources have been going on for many years now, but they still reserve a few surprises, as peculiar sources are still found today, several years after the publication of the first IBIS and BAT catalogues (e.g. Masetti et al. 2007). Follow-up campaigns at different wavelengths are not only paramount in determining the true nature of new discoveries, but are also essential to resolve ambiguities in source identification, as is the case for the sources presented in this paper. In this work we aim at unveiling the true nature of these three sources, IGR J12134-6015, IGR J16058-7253 and Swift J2037.2+4151, for which their classification is still unclear. IGR J12134-6015 and Swift J2037.2+4151 have both been classified as beamed AGN in several BAT catalogues, but left unclassified in various INTEGRAL surveys, while through a multi-band approach we propose a different classification. As for the third source analysed here, IGR J16058-7253, the issue arises not in the classification of this object as an AGN, but rather on the origin of the high energy emission, this object being a blending of two different active galaxies. Here we collect all previous and new X-ray data (Swift/XRT, Chandra and NuSTAR) and re-analyse them adding the available information at other wavelengths.

\section{X-RAY DATA REDUCTION}

Swift/XRT observed all the sources studied here; these data have been previously presented by Landi et al. (2011a), however we re- 
Table 1. Observation log of the sources analysed here.

\begin{tabular}{|c|c|c|c|c|c|c|}
\hline Source & $\mathbf{R A}$ & Dec & Pos. error & Telescope & Obs. date & Exp. (ksec) \\
\hline \multirow[t]{4}{*}{ IGR J12134-6015 } & $12^{\mathrm{h}} 13^{\mathrm{m}} 24.00^{\mathrm{s}}$ & $-60^{\mathrm{d}} 15^{\mathrm{m}} 16.541^{\mathrm{s}}$ & $0.64^{\prime \prime}$ & Chandra (HRC-I) & $26 / 02 / 2011$ & 1.17 \\
\hline & & & & XRT & 06/02/2011 & 2.86 \\
\hline & & & & XRT & 09/02/2011 & 3.78 \\
\hline & & & & XRT & $11 / 02 / 2011$ & 2.5 \\
\hline \multirow{6}{*}{$\begin{array}{c}\text { IGR J16058-7253* } \\
\text { (LEDA 259433) }\end{array}$} & $16^{\mathrm{h}} 05^{\mathrm{m}} 22.8^{\mathrm{s}}$ & $-72^{\mathrm{d}} 53^{\mathrm{m}} 55.3^{\mathrm{s}}$ & $3.8^{\prime \prime}$ & XRT & $15 / 04 / 2009$ & 2.23 \\
\hline & & & & XRT & $31 / 12 / 2009$ & 7.28 \\
\hline & & & & XRT & $10 / 04 / 2010$ & 5.14 \\
\hline & & & & XRT & $11 / 05 / 2010$ & 1.73 \\
\hline & & & & XRT & $12 / 05 / 2010$ & 0.66 \\
\hline & & & & NuSTAR & $01 / 03 / 2019$ & 22.2 \\
\hline \multirow{6}{*}{$\begin{array}{c}\text { IGR J16058-7253 } \\
\text { (LEDA 259580) }\end{array}$} & $16^{\mathrm{h}} 06^{\mathrm{m}} 06.7^{\mathrm{s}}$ & $-72^{\mathrm{d}} 52^{\mathrm{m}} 40.6^{\mathrm{s}}$ & $4.1^{\prime \prime}$ & XRT & $15 / 04 / 2009$ & 2.23 \\
\hline & & & & XRT & $31 / 12 / 2009$ & 7.28 \\
\hline & & & & XRT & $10 / 04 / 2010$ & 5.14 \\
\hline & & & & XRT & $11 / 05 / 2010$ & 1.73 \\
\hline & & & & XRT & $12 / 05 / 2010$ & 0.66 \\
\hline & & & & NuSTAR & $01 / 03 / 2019$ & 22.2 \\
\hline \multirow[t]{3}{*}{ Swift J2037.2+4151 } & $20^{\mathrm{h}} 37^{\mathrm{m}} 05.5^{\mathrm{s}}$ & $41^{\mathrm{d}} 50^{\mathrm{m}} 05^{\mathrm{s}}$ & $3.5^{\prime \prime}$ & XRT & $17 / 08 / 2006$ & 5.39 \\
\hline & & & & XRT & $17 / 12 / 2006$ & 4.87 \\
\hline & & & & XRT & $04 / 12 / 2007$ & 2.20 \\
\hline
\end{tabular}

Notes: ${ }^{\dagger}$ : Chandra coordinates; ${ }^{\ddagger}$ XRT coordinates

analysed them together with other multiwavelength gathered specifically for this work. Swift/XRT data reduction was performed using the standard data pipeline package (XRTPIPELINE v. 0.13.2) in order to produce screened event files (see Landi et al. 2010). Source events were extracted within a circular region with a radius of 20 pixels (1 pixel corresponding to $2.36 \mathrm{arcsec}$ ) centred on the source position, while background events were extracted from a source-free region close to the X-ray source of interest. The spectra were obtained from the corresponding event files using the XSELECT v. 2.4c software. We used version v.014 of the response matrices and created individual ancillary response files using the task xrtmkarf v.0.6.3.

Chandra HRC-I data reduction for IGR J12134-6015 was performed using CIAO-4.13 and CALDB_4.9.4; the source coordinates have been obtained with the task wavdetect, which returns the source position; as for the positional uncertainty, we assume the nominal one of 0.64 arcsec as done by Karasev et al. (2012).

NuSTAR data for IGR J16058-7253 were reduced using the nustardas_01Apr120_V1.9.2 and CALDB version 20200429. Spectral extraction and the subsequent production of response and ancillary files was performed using the nuproducts task with an extraction radius of 50"; to maximise the signal-to-noise ratio $(\mathrm{S} / \mathrm{N})$, the background spectrum was extracted from a $70^{\prime \prime}$ radius circular region as close to the source as possible.

All spectra were binned with grppha in order to achieve a minimum of 20 counts per bin, in order to apply the $\chi^{2}$ statistics and spectral fitting was performed in XSPEC v12.11.1 (Arnaud 1996); uncertainties are listed at the $90 \%$ confidence level $\left(\Delta \chi^{2}=2.71\right.$ for one parameter of interest). Abundances were all set to Solar and the cross-sections employed are photoelectric ones.

\section{IGR J12134-6015}

IGR J12134-6015 was first reported as a high energy emitting source by Krivonos et al. (2010), in their 7-year INTEGRAL all sky survey; these authors suggested that IGR J12134-6015 is associated with the ROSAT source 1RXS J121324.5-601458. The source was also listed in the BAT 58-month and 70-month catalogue (Baumgartner et al. 2010, 2013), where its likely counterpart was identified as the same ROSAT source, but no firm identification was reported. The BAT 105-month (Oh et al. 2018) and the latest BAT 157month (https://swift.gsfc.nasa.gov/results/bs157mon/) catalogues instead classified this source as a beamed AGN, as also did Krivonos et al. 2012 in their 9-year INTEGRAL survey, whereas in Bird et al. (2016) the source is detected in a 1605.1 day outburst starting on MJD 53292.6, at a $6.3 \sigma$ level (maximum significance during the outburst), suggesting variability at high energy but no classification is given.

The first X-ray follow-up of IGR J12134-6015 was carried out by both Landi et al. (2011a) and Karasev et al. (2012), who identified the 2MASS counterpart and confirmed the association with the ROSAT source; Landi et al. (2011a) also found, coincident with this object, the XMM Slew source XMMSL1 J121323.5-601517. Spectral properties derived from Swift/XRT data by Landi et al. (2011a) pointed to a Galactic nature for this source, whereas Karasev et al. (2012) suggested that the source might be an extragalactic object, raising the question of what its true nature might be.

The extragalactic nature of this object is also challenged in a paper by Paliya et al. (2019), where the authors study the physical properties of blazars extracted from the BAT 105-month catalogue; however, the authors exclude IGR J12134-6015 from the list of BAT 
blazars, on the basis of its broad-band properties, which are not typical of beamed AGN.

In order to reach firmer conclusions on the true nature of IGR J12134-6015, we investigated further this source at multiple wavelengths, starting with all the available X-ray measurements. Apart from the Swift/XRT data analysed by Landi et al. (2011a), IGR J12134-6015 has been observed once by the HRC-I instrument onboard Chandra (see Table 1). Analysis of the $0.8-10 \mathrm{keV}$ full band image (see Fig. 1) confirms that there is only one X-ray source in the field of view, at the coordinates reported in Table 1 and consistent with the position of the ROSAT counterpart. Within 4 arcsec of the Chandra position, we find two Gaia sources listed in the Early Data Release 3 archive (Brown et al. 2021), but only one is coincident with the X-ray source detected by Chandra (see Fig. 1), while the other lies too far away from the Chandra positional error circle. The Gaia counterpart of IGR J12134-6015, Gaia 6058696067111698560 at 0.37 arcsec from the Chandra position, has coordinates RA = $12^{\mathrm{h}} 13^{\mathrm{m}} 23.95^{\mathrm{s}}$ and $\mathrm{Dec}=-60^{\mathrm{d}} 15^{\mathrm{m}} 16.8^{\mathrm{s}}$ and magnitudes $\mathrm{G}=18.77$ $(\mathrm{S} / \mathrm{N}=13), \mathrm{G}_{\mathrm{BP}}=18.01(\mathrm{~S} / \mathrm{N}=4)$ and $\mathrm{G}_{\mathrm{RP}}=17.01(\mathrm{~S} / \mathrm{N}=5)$; the parallax is $1.471 \pm 0.06$ mas, yielding an estimated absolute magnitude of $\sim 9.6^{1}$ and a distance of of 674 pc (Bailer-Jones et al. 2021), indeed suggesting a Galactic source.

Considering the Gaia colours for this source and following the diagram reported by Eyer et al. (2019) (see Fig. 2 in their paper), IGR J12134-6015 falls in the region just below the Main Sequence occupied by dwarfs and sub-dwarfs. This is also supported by the diagram shown in Fig. 2 of Abril et al. (2020), where again IGR J121346015 occupies a region consistent with both CVs and Dwarf Novae. Particularly, IGR J12134-6015 lies in the region where short-period Polars are found, well inside the range of $\mathrm{G}_{\mathrm{BP}}-\mathrm{G}_{\mathrm{RP}}$ and absolute $\mathrm{G}$ magnitude identified for CVs by these authors. The Gaia counterpart of IGR J12134-6015 is also listed in the ASAS-SN catalog (Jayasinghe et al. 2018 and Jayasinghe et al. 2020) of variable stars, with a parallax consistent with the Gaia measurements, again pointing to a Galactic nature for IGR J12134-6015. The optical light curve, found on the ASAS-SN on-line database ${ }^{2}$, shows an outburst of about 1 magnitude at the very beginning of the ASAS-SN coverage, with the amplitude compatible with that of a dwarf nova.

\subsection{Optical/NIR spectroscopy}

Two spectra over the optical/near-infrared (NIR) range were collected at ESO-VLT with XShooter (covering the 3000-25000 A, range; see Vernet et al. 2011 for details on the instrument) on Jan 17 and 18, 2019, under the ESO programme 0102.D-0918(A) - PI: S. Chaty - with exposure times of 556, 1584 and $2479 \mathrm{~s}$ in the blue, visual and NIR arms, respectively.

We retrieved the pipeline-analysed, wavelength and flux calibrated spectra for each day from the ESO Science Portal ${ }^{3}$; given that the overall shape was comparable, we stacked the spectra together to increase the $\mathrm{S} / \mathrm{N}$.

The combined spectrum (Fig. 2) appears to be noisy, with most of the signal at longer (NIR) wavelengths where wide atmospheric bands are apparent, and an increase in flux towards the blue range.

\footnotetext{
1 To estimate the absolute magnitude we used the formula $\mathrm{M}_{\mathrm{G}}=$ $\mathrm{m}_{\mathrm{g}}+5+5 \log (\varpi / 1000)$, where $\varpi$ is the source parallax (Babusiaux et al. 2018).

2 https://asas-sn.osu.edu/variables/324039

3 http://archive.eso.org/scienceportal/home
}



Figure 1. Chandra $0.8-10 \mathrm{keV}$ HRC-I image of the sky region around IGR J12134-6015. The green circle is centered on the Chandra coordinates and represents their positional error of $0.64 \operatorname{arcsec}$ (see Karasev et al. 2012). The magenta cross is the likely GAIA counterpart of the X-ray source, while the yellow cross is the second nearby source found in the GAIA catalogue.

Also, $\mathrm{H}_{\alpha}$ and $\mathrm{H}_{\beta}$ lines (and possibly $\mathrm{H}_{\gamma}$ ) are detected in emission at redshift zero with fluxes $(8.5 \pm 0.9) \times 10^{-16},(2.6 \pm 0.8) \times 10^{-16}$ and $\approx 2 \times 10^{-16} \mathrm{erg} \mathrm{cm}^{-2} \mathrm{~s}^{-1}$, respectively. An indication of extended wings is present in the $\mathrm{H}_{\alpha}$ emission profile (see the zoom-in on the $\mathrm{H}_{\alpha}$ region in Fig. 3), while the $\mathrm{S} / \mathrm{N}$ is too low in the case of $\mathrm{H}_{\beta}$ to perform a similar investigation. These findings confirm that the object belongs to the Galaxy and that it may well be an accreting system composed of a low mass, late spectral type dwarf star (which dominates the emission in the NIR) losing mass onto a compact object with an accretion structure around it, possibly a disk, which mostly emits in the bluer parts of the XShooter spectrum. Also, because of the detection of short-wavelength photons, the system should not suffer from extreme reddening and thus should not be very far from Earth: this consideration on the distance is supported by the Gaia data.

If we take the Gaia distance and use the $2-10 \mathrm{keV}$ band flux of $5 \times$ $10^{-12} \mathrm{erg} \mathrm{cm}^{-2} \mathrm{~s}^{-1}$ reported by Landi et al. (2011b) (see also next section), we find that the X-ray output of the source is few times $10^{32} \mathrm{erg} \mathrm{s}^{-1}$, which places IGR J12134-6015 well within the X-ray luminosity range of CVs observed with INTEGRAL (see e.g. Landi et al. 2009. Moreover, as can be seen in Figure 6 of De Martino et al. (2020), the source might occupy the low-luminosity end of the space where this class of objects is located. Moreover, the optical absolute magnitude of the source determined from the Gaia data is as well typical of this kind of source $(\approx+9$; see e.g. Warner 1995).

\subsection{X-ray analysis}

XRT observed IGR J12134-6015 shortly on four occasions in February 2011, but as the second observation was $\sim 200 \mathrm{sec}$ long, we reduced only the longer ones (one on the $6^{\text {th }}$, which we call Observa- 




Figure 2. Optical-NIR XShooter spectrum of the counterpart of IGR J12134-6015 rebinned at $6 \AA$ ( $~ 10$ pixels). The NIR telluric features are indicated with the symbol $\oplus$. Apart from $\mathrm{H}_{\alpha}$, all narrow features readily visible in the spectrum are due to noise.

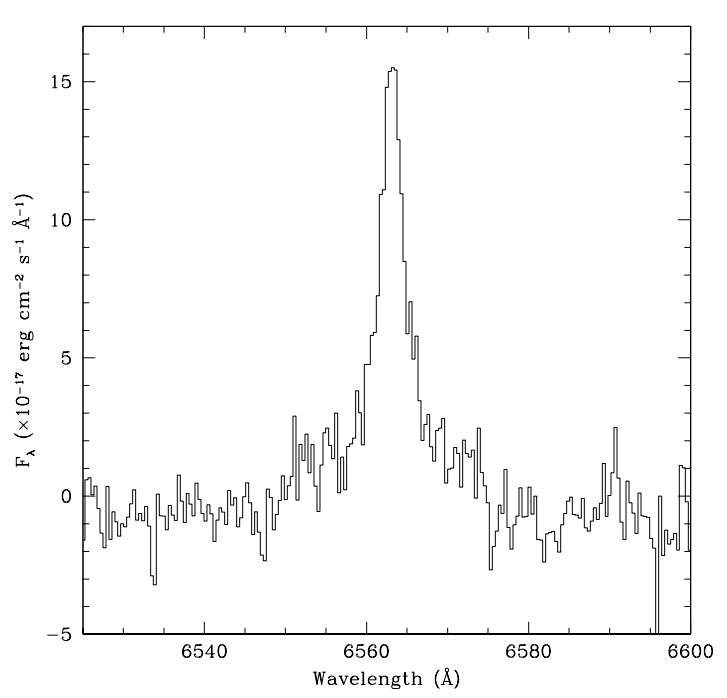

Figure 3. Zoom-in on the $\mathrm{H}_{\alpha}$ region of the optical spectrum of IGR J121346015.

tion 1 , one on the $9^{\text {th }}$, which we call Observation 2 , and the last one on $11^{\text {th }}$, called Observation 3; see Table 1). The source is not very bright and its detection level is quite low $(14.7 \sigma$ for Observation 1, $22.4 \sigma$ for Observation 2 and $12.3 \sigma$ for Observation 3). Unfortunately, while there are Chandra HRC-I data available, no data from the ACIS instrument are present in the archive, so we could not extract a spectrum from the Chandra data.

$X$-ray variability. Since our multiwavelength data strongly suggest that IGR J12134-6015 is a Cataclysmic Variable, a class of objects which is characterised by variability both on short and on long timescales, we did a quantitative analysis to see whether variability is present in our X-ray observations (a thorough timing analysis is beyond the scope of the present paper). In order to verify if variability on short timescales (less than an hour) is present, we analysed the XRT light curves in each of the three observing periods, using the lcstats tool within the ftools, which returns the constant source probability, associated to the Chi-square value, therefore providing evidence of variability. The analysis of the light curve relative to Observation 1 suggests that during this period the source has remained more or less constant (with a probability of constancy of $\sim 0.82$ ); analysis of the light curves for the subsequent two periods instead points at some degree of variability, having a probability of constancy of $0.37 \times 10^{-17}$ for Observation 2 and of $0.24 \times 10^{-12}$ for Observation 3. Comparing the fluxes of the three observations (see Table 2), we find that flux variability (up to a factor of 2) is indeed present on timescales of days. From the values reported in Table 2, it is evident that the source has changed from a low state in the first observation, to a high state in the second and then returned to a low state in the last one, all in a matter of a few days. This is also confirmed by the XMM Slew 0.2-12 keV flux measured a few years prior (in 2007), where the source was in a low state with a flux of $2.41 \times 10^{-12} \mathrm{erg} \mathrm{cm}^{-2} \mathrm{~s}^{-1}$ consistent with the source state in Observation 1 and 3, but not with Observation 2 where the flux is twice as high as in the other two observations.

In order to assess variability in the hard X-rays, we ran the task lcstats on the 157-month Crab-weighted BAT light curve. The statistical analysis indeed suggests that some degree of variability (possibly on time scales of months) is also seen at higher energies, since the probability of constancy is $0.38 \times 10^{-2}$. Variability is therefore found in a wide range of timescales, as is expected in systems where accretion onto a white dwarf takes place; these systems are in 
Table 2. Spectral parameters for IGR J12134-6015. Model employed is phabs*po.

\begin{tabular}{|c|c|c|c|c|}
\hline Obs. & $\begin{array}{c}\mathrm{N}_{\mathrm{H}} \\
\mathrm{cm}^{-2}\end{array}$ & $\Gamma$ & 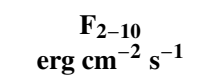 & $\chi^{2}$ (d.o.f.) \\
\hline 1 & $<0.27 \times 10^{22}$ & $1.03_{-0.26}^{+0.44}$ & $\left(4.12_{-1.01}^{+2.73}\right) \times 10^{-12}$ & $9.22(6)$ \\
\hline 2 & $>0.82 \times 10^{22}$ & $0.85 \pm 0.15$ & $\left(8.03_{-1.18}^{+1.22}\right) \times 10^{-12}$ & 39.83 (19) \\
\hline 3 & $>0.82 \times 10^{22}$ & $1.35_{-0.59}^{+0.71}$ & $\left(4.46_{-2.46}^{+6.84}\right) \times 10^{-12}$ & $2.46(3)$ \\
\hline
\end{tabular}

fact characterised by variability on different time-scales and across multiple wavelengths.

$X$-ray spectral analysis. As shown above, the source has a certain degree of variability in flux and given that also variability in spectral shape cannot be excluded, we have analysed each of the three observations separately and then we fitted the sum of the three spectra, in order to have an average spectrum with higher statistics.

The spectra of the three observations were first fitted with a simple model (phabs*po in XSPEC terminology) and since the Gaia data suggests that the source is very near, we left the column density free to vary, without adding any Galactic $\mathrm{N}_{\mathrm{H}}$ in order not to over-estimate the absorption along the line of sight. This model does not describe sufficiently well the data, since the reduced $\chi^{2}$ are $1.54,2.10$ and 0.82 for Observation 1, 2 and 3 respectively (see Table 2). However, as can be seen from Table 2, our spectra are quite poor from a statistical point of view, except for Observation 2, to allow for the use of more complex models. For this reason, we attempted to use a more physical model only for data relative to Observation 2. To the simple power-law component, we added a thermal one, in the form of either a blackbody or a bremsstrahlung (see e.g. Bernardini et al. 2012 and Bernardini, de Martino, Falanga, Mukai, Matt, BonnetBidaud, Masetti \& Mouchet dem). From the fits reported in Table 3, it can be seen that the model that best describes the data is the one where the thermal component is in the form of a bremsstrahlung, whereas the model with the blackbody component returns values for the temperature which are too high to have any physical meaning and are also not well constrained. The addition of a power-law component in our fits, although not physically meaningful, is used as an approximation to highlight an underlying spectral complexity at soft energies, which with the current data we are not able to fit with more appropriate and physical models. We also point out that the fact that the values for the absorbing column density found in our analyis differ slightly from the previous values reported by both Landi et al. (2011a) and Karasev et al. (2012); this could be due to the fact that while these authors used a summed spectrum of the three observations, here we consider each observation separately and only afterwards the sum of the three. This can lead, due to the poorer statistics of single observations, to larger errors and /or discrepancies in values. It is therefore evident that in order to have a better understanding of the X-ray behaviour of this source, high quality spectra are needed, making IGR J12134-6015 the ideal target for future observations with X-ray facilities such as XMM-Newton or Chandra.

Since nothing can be deduced from the available spectra regarding spectral variability, we opted to boost the statistics of our data by summing the three data sets and fitting the resulting average spectrum. The results are shown in Table 3; the fit is not acceptable as evident from the $\chi^{2}$ value and although the bremsstrahlung temperature is well constrained, some residuals are still present (see Figure 4). This could be an indication that some degree of spectral variability is present in the source, however our data are not statistically
Table 3. Spectral parameters for thermal models of IGR J12134-6015.

\begin{tabular}{|c|c|c|c|c|}
\hline \multicolumn{5}{|c|}{ Observation 2, phabs* (po+bb). } \\
\hline $\begin{array}{c}\mathrm{N}_{\mathrm{H}} \\
\mathbf{c m}^{-2}\end{array}$ & $\Gamma$ & $\begin{array}{c}\text { kT } \\
\text { keV }\end{array}$ & $\begin{array}{c}F_{2-10} \\
\operatorname{erg~cm}^{-2} \mathrm{~s}^{-1}\end{array}$ & $\chi^{2}$ (d.o.f.) \\
\hline$>0.82 \times 10^{22}$ & $2.07_{-0.74}^{+0.95}$ & $2.75_{-0.93}^{+35.61}$ & $\left(8.95_{-0.24}^{+0.24}\right) \times 10^{-12}$ & $27.61(17)$ \\
\hline \multicolumn{5}{|c|}{ Observation 2, phabs* (po+bremss). } \\
\hline $\begin{array}{c}\mathrm{N}_{\mathrm{H}} \\
\mathrm{cm}^{-2}\end{array}$ & $\Gamma$ & $\begin{array}{l}\mathrm{kT} \\
\mathrm{keV}\end{array}$ & $\begin{array}{c}F_{2-10} \\
\operatorname{erg~cm}^{-2} \mathrm{~s}^{-1}\end{array}$ & $\chi^{2}$ (d.o.f.) \\
\hline$>0.82 \times 10^{22}$ & $0.74 \pm 0.16$ & $0.06_{-0.04}^{+0.06}$ & $\left(8.59_{-1.50}^{+1.54}\right) \times 10^{-12}$ & $17.81(17)$ \\
\hline \multicolumn{5}{|c|}{ Average Spectrum, phabs* (po+bremss). } \\
\hline $\begin{array}{c}\mathrm{N}_{\mathrm{H}} \\
\mathrm{cm}^{-2}\end{array}$ & $\Gamma$ & $\begin{array}{l}\text { kT } \\
\text { keV }\end{array}$ & $\begin{array}{c}F_{2-10} \\
\operatorname{erg~cm}^{-2} \mathrm{~s}^{-1}\end{array}$ & $\chi^{2}$ (d.o.f.) \\
\hline$>0.82 \times 10^{22}$ & $0.79 \pm 0.11$ & $0.109_{-0.04}^{+0.07}$ & $\left(6.55_{-0.89}^{+0.87}\right) \times 10^{-12}$ & $66.97(36)$ \\
\hline
\end{tabular}

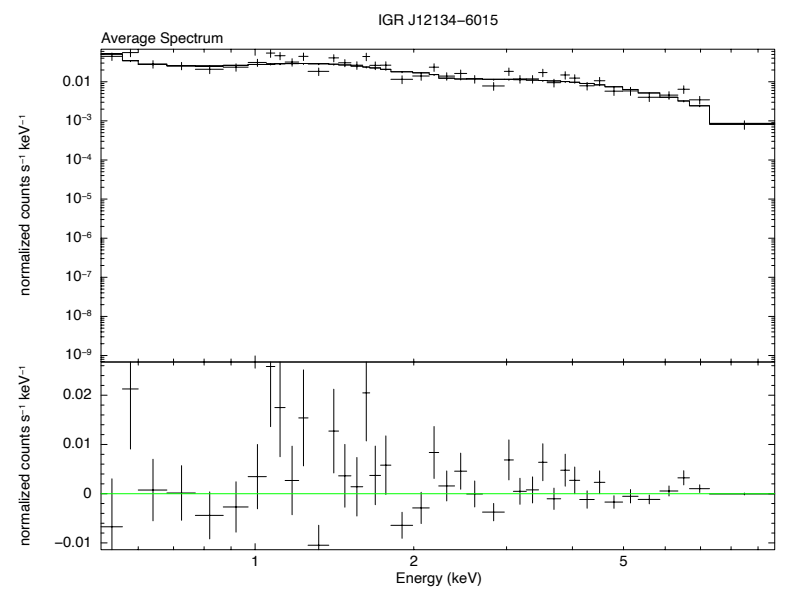

Figure 4. $0.5-9.5 \mathrm{keV}$ average spectrum of IGR J12134-6015, the model employed is phabs*(po+bremss)

good enough to actually highlight changes in the spectral parameters. For this reason, the summed spectrum must be carefully considered and also does not allow to perform a broad-band spectral analysis employing the available INTEGRAL/IBIS and Swift/BAT spectra, since the high energy data are averaged over very long time periods.

We can however analyse the high energy spectra from INTEGRAL/IBIS and Swift/BAT and compare them, in order to assess the source behaviour above $20 \mathrm{keV}$. We fit the $20-100 \mathrm{keV}$ spectra together using a simple power-law (leaving the photon index and the normalisation untied for the two spectra) multiplied by the cflux component so to have an estimate on the fluxes and their errors (see Table 4). Clearly the spectra, which we remind are time-averaged, are very different from one another, a possible indication of variability even at high energies. In addition, we point out that the INTEGRAL/IBIS spectrum is averaged over data taken up to 2013, while the BAT one extends further in time (to about 2018), suggesting that the great difference in the two spectra is indeed due to the fact that the source has periods of low and high states which are affecting 
Table 4. High energy spectral parameters for IGR J12134-6015. Model employed is cflux*po.

\begin{tabular}{|c|c|c|}
\hline Instr. & $\Gamma$ &  \\
\hline IBIS & $2.06_{-0.73}^{+1.00}$ & $\left(5.88_{-2.20}^{+2.29}\right) \times 10^{-12}$ \\
\hline BAT & $4.98_{-0.11}^{+0.06}$ & $\left(3.89_{-0.17}^{+0.16}\right) \times 10^{-12}$ \\
\hline
\end{tabular}

also the high energy, as already highlighted in the timing analysis (see above).

Taking into account the evidence provided by our multiwavelength approch, we can conclude that IGR J12134-6015 is a Galactic object: there is evidence of X-ray variability both on short (days) and long (months, years) timescales, and the optical/NIR and X-ray spectral data analysis, combined with the inferred distance, strongly suggests that IGR J12134-6015 is a CV.

\section{IGR J16058-7253}

IGR J16058-7253 was first listed as a high energy emitting source in 2010 in the INTEGRAL 7-year all sky survey by Krivonos et al. 2010, where it was tentatively associated with the IR source IRAS F15596-7245. In the BAT 58-month (Baumgartner et al. 2010) and in the 70-month (Baumgartner et al. 2013) catalogues, the source is associated with the same IR counterpart, but listed as 2MASX $\mathrm{J} 16052330-7253565$ and for the first time a classification as a galaxy is provided. The likely counterpart of IGR J16058-7253 was more firmly identified thanks to the Swift/XRT follow-up observation performed by Landi et al. (2011a) which detected two sources in the Swift/XRT field of view, located within the INTEGRAL/IBIS and Swift/BAT positional uncertainties. These two X-ray sources were subsequently identified by Masetti et al. (2013) as two AGN and classified as LEDA 259580, a Seyfert 2 at $\mathrm{z}=0.09$, and LEDA 259433, a likely Seyfert 2 at $z=0.069$ (see in the following). In the latest INTEGRAL/IBIS survey (Bird et al. 2016), IGR J16058-7253 was associated to both AGN, which lie just 3.4 arcmin apart and could not be resolved by IBIS (whose angular resolution is about 12 arcmin) as the positional accuracy is of the order of 4 arcmin. However, in the two latest BAT catalogues, the 105-month (Oh et al. 2018)) and the 157-month (https://swift.gsfc.nasa. gov/results/bs157mon/) the likely counterpart of IGR J160587253 was identified as the Seyfert 2 galaxy LEDA 259433. In a subsequent work by Bär et al. 2019, IGR J16058-7253 is classified as an ultra-luminous AGN, characterised by both high bolometric $\left(\log \mathrm{L}_{\mathrm{bol}}=45.61 \mathrm{erg} \mathrm{s}^{-1}\right)$ and high hard X-ray $\left(\log \mathrm{L}_{14-195}=44.71 \mathrm{erg}\right.$ $\mathrm{s}^{-1}$ ) luminosities.

The discrepancy in associations between the BAT and IBIS catalogues is mainly due to the positions and relative uncertainties derived by the two instruments. The BAT catalogue reports a position for IGR J16058-7253 at RA $=16^{\mathrm{h}} 05^{\mathrm{m}} 23.28^{\mathrm{s}}$ and Dec $=-$ $72^{\mathrm{d}} 53^{\mathrm{m}} 56.4^{\mathrm{s}}$, with an error circle of 3 arcmin radius; within this error circle, only LEDA 259433 is found, while LEDA 259580 lies just outside, hence the association proposed in the BAT catalogues. Bird et al. 2016 instead found that the source is positioned at RA = $16^{\mathrm{h}} 05^{\mathrm{m}} 52.8^{\mathrm{s}}$ and Dec $=-72^{\mathrm{d}} 54^{\mathrm{m}} 00^{\mathrm{s}}$, with an error circle of 4 arcmin radius encompassing both sources, with the two of them correctly listed as likely associations. As a result, at least in the case of the IBIS detection and possibly also for the BAT one, it is not possible

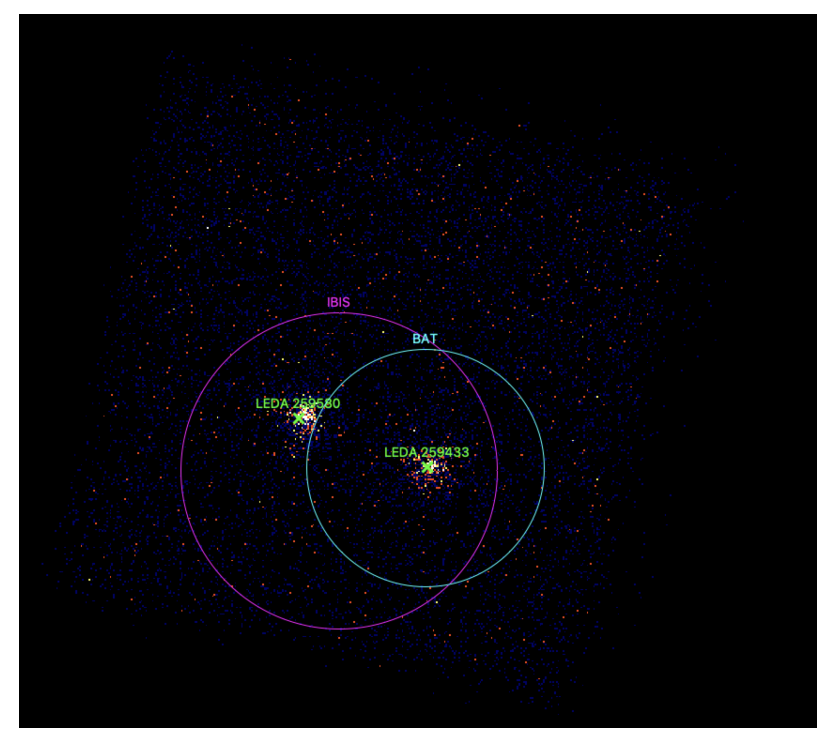

Figure 5. 10-80 keV NuSTAR image of the sky region containing the two counterparts of IGR J16058-7253. The BAT 3 arcmin error circle is shown in cyan, together with the IBIS 4 arcmin error circle shown in magenta.

to exclude that both sources contribute to the high energy emission above $20 \mathrm{keV}$.

As is often the case with likely counterparts of high energy sources and particularly when an ambiguity is present, X-ray data are essential in identifying the correct counterpart. IGR J16058-7253 has been observed by both Swift/XRT (between 2009 and 2010) and more recently by NuSTAR (in 2019), which, given its imaging capability at high energy, can be valuable in determining if only one or both sources emit at energies greater than $10 \mathrm{keV}$. To this aim, we have performed imaging analysis of NuSTAR data by selecting high energy photons ( $>10 \mathrm{keV})$, and indeed inspection of the $10-80 \mathrm{keV}$ NuSTAR image (see Fig. 5), clearly shows that LEDA 259433 and LEDA 259580 are both detected.

For these reasons, we believe that is not possible to disentangle the hard X-ray emission from the two sources neither with INTEGRAL, nor with BAT. Consequently it is not possible to attribute the measured flux to a single source based on either INTEGRAL or BAT measurements and therefore the estimate of the bolometric and Eddington luminosities for IGR J16058-7253 given by Bär et al. (2019) and based solely on the BAT flux is not correct.

\subsection{X-ray spectral analysis}

In the following we provide a detailed spectral analysis, by fitting combined XRT/NuSTAR spectra of LEDA 259433 and LEDA 259580.

For each source, we fit the XRT and NuSTAR spectra together, employing a simple phenomenological model, given the poor quality of the data, consisting of a simple power-law absorbed by Galactic and intrinsic absorption (our baseline model, const*phabs*phabs*po in XSPEC terminology). In the two fits we also added a cross-calibration constant to account for mismatches in the calibration between XRT and NuSTAR and also to account for flux variability, given the large time-span between the two sets of observations.

LEDA 259433. The XRT/NuSTAR broad-band spectrum of the likely Seyfert 2 LEDA 259433 covers the $2-50 \mathrm{keV}$ range, as there is not enough statistics below $2 \mathrm{keV}$ and above $50 \mathrm{keV}$ to have a 


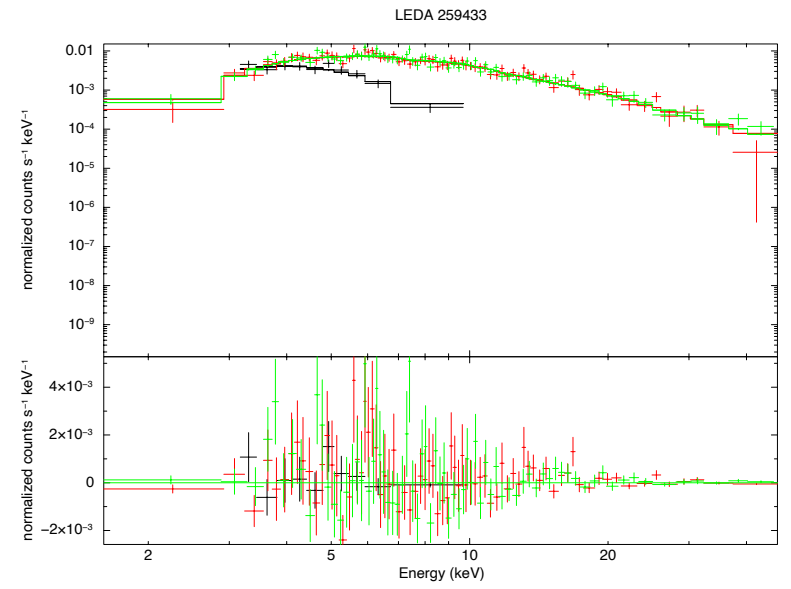

Figure 6. XRT/NuSTAR 2-50 keV broad-band spectrum of LEDA 259433. The model employed is const*phabs*phabs*po.

spectrum in a broader energy range. The data are well fitted by our baseline model, with a $\chi^{2}$ of 142.90 for 155 d.o.f., resulting in a $\Delta \chi^{2}$ of 0.92 (see Fig. 6). We find an $\mathrm{N}_{\mathrm{H}}$ of $\left(9.43_{-1.54}^{+2.05}\right) \times 10^{22} \mathrm{~cm}^{-2}$ (see Table 5), therefore suggesting that the source might indeed be a type 2 AGN as suggested in previous works (see e.g. Landi et al. 2011a and Masetti et al. 2013). The photon index is $1.56 \pm 0.09$, while the cross-calibration constants between XRT and the two NuSTAR detectors are around 0.9 and 1 respectively. As suggested by the values of the constants, the source does not show signs of flux variability, as the $2-10 \mathrm{keV}$ XRT flux is $2.32 \times 10^{-12} \mathrm{erg} \mathrm{cm}^{-2} \mathrm{~s}^{-1}$, while the NuSTAR/FPMA $2-10 \mathrm{keV}$ flux is $2.05 \times 10^{-12} \mathrm{erg} \mathrm{cm}^{-2} \mathrm{~s}^{-1}$. The $20-100$ $\mathrm{keV}$ flux is $9.45 \times 10^{-12} \mathrm{erg} \mathrm{cm}^{-2} \mathrm{~s}^{-1}$ for NuSTAR/FPMA (the FPMB flux is fully compatible with this value).

LEDA 259580. As for LEDA 259580, the broad-band fit is performed again in the $2-50 \mathrm{keV}$ range, due to the statistical quality of our data. The baseline model fits fairly well the data, having a $\chi^{2}$ of 171.03 for 149 d.o.f., resulting in a $\Delta \chi^{2}=1.15$ (see Fig. 7 and Table 5). The intrinsic column density is $\mathrm{N}_{\mathrm{H}}=\left(25.30_{-4.31}^{+4.87}\right) \times 10^{22} \mathrm{~cm}^{-2}$, consisting with the type 2 AGN nature of this source, and the photon index is $\Gamma=1.48 \pm 0.12$, while the cross calibration constants are a bit higher than 1 (around 1.5), suggesting that the source might have undergone some changes in its flux. Indeed the $2-10 \mathrm{keV}$ XRT flux is found to be $1.15 \times 10^{-12} \mathrm{erg} \mathrm{cm}^{-2} \mathrm{~s}^{-1}$, while the NuSTAR/FPMA flux is $1.77 \times 10^{-12} \mathrm{erg} \mathrm{cm}^{-2} \mathrm{~s}^{-1}$, indicative of minor flux variability. The $20-100 \mathrm{keV}$ flux is instead $1.57 \times 10^{-11} \mathrm{erg} \mathrm{cm}^{-2} \mathrm{~s}^{-1}$ for NuSTAR/FPMA (the value is similar also for the FPMB detector).

From the fluxes extrapolated from the spectral fits, we can attempt to draw some conclusions on the hard X-ray emission of these sources. If we take the NuSTAR $20-100 \mathrm{keV}$ fluxes measured from our fits, we find that their sum is $2.51 \times 10^{-11} \mathrm{erg} \mathrm{cm}^{-2} \mathrm{~s}^{-1}$, consistent with the flux obtained from the IBIS spectrum of $2.21 \times 10^{-11} \mathrm{erg}$ $\mathrm{cm}^{-2} \mathrm{~s}^{-1}$, but higher than the flux measured from the BAT spectrum of $1.63 \times 10^{-11} \mathrm{erg} \mathrm{cm}^{-2} \mathrm{~s}^{-1}$. From the fluxes reported in Table 5 it is also evident that LEDA 259580, which was not identified as one of the possible counterparts of IGR J16058-7253 in the BAT catalogues, appears to be the dominant source above $20 \mathrm{keV}$. Regarding LEDA 259433 , from the $2-10 \mathrm{keV}$ flux, we can estimate the luminosity in this band, which we found to be $3.14 \times 10^{43} \mathrm{erg} \mathrm{s}^{-1}$. From this quantity, we calculated the bolometric luminosity employing the correction proposed by Marconi et al. (2004) and we find it to be



Figure 7. XRT/NuSTAR 2-50 keV broad-band spectrum of LEDA 259580. The model employed is const*phabs*phabs*po.

$7.41 \times 10^{44} \mathrm{erg} \mathrm{s}^{-1}$, in perfect agreement with the median of bolometric luminosities for type 2 AGN found by Lusso et al. (2012). Assuming the black hole mass of $7.08 \times 10^{7} \mathrm{M}_{\odot}$ reported by Bär et al. $(2019)^{4}$ for this source, we found that the Eddington luminosity is $8.92 \times 10^{45} \mathrm{erg} \mathrm{s}^{-1}$, leading to an Eddington ratio of 0.083 , again fully consistent with the median value for type 2 AGN reported by Lusso et al. (2012), therefore excluding the possibility of this source being an ultra-luminous AGN.

\section{SWIFT J2037.2+4151}

Swift J2037.2+4151 was first reported as a high energy source by Tueller et al. (2006) and later confirmed in Jem-X pointings by Westergaard et al. (2006). The source was also listed as a transient object in the BAT 58-month and 70-month catalogues (Baumgartner et al. 2010, 2013); in the BAT 105-month (Oh et al. 2018) and 157month (https://swift.gsfc.nasa.gov/results/bs157mon/) catalogues, the source is associated with the IR counterpart SSTSL2 J203705.58+415005.3 and classified as a beamed AGN. However in Paliya et al. 2019, the source is excluded from the list of BAT blazars on the basis of its broad-band characteristics. Swift J2037.2+4151 was also listed in several IBIS surveys: in the 7-year and 9-year surveys by Krivonos et al. $(2010,2012)$ and in the latest INTEGRAL/IBIS catalogue by Bird et al. (2016), where it was listed as a transient source; in all these INTEGRAL catalogues, no clear classification was given. The X-ray counterpart of Swift J2037.2+4151 was identified through Swift/XRT follow-up observations by Landi et al. (2011a) (see Table 1 for the XRT coordinates and positional error) and was associated to a 2 MASS source, coincident with the NIR counterpart proposed by BAT, which is quite bright and has magnitudes $\mathrm{J}=16.167 \pm 0.088, \mathrm{H}=13.456 \pm 0.033$ and $\mathrm{K}=12.128 \pm 0.020$. We have investigated other multiwavelength catalogues and found

\footnotetext{
${ }^{4}$ BH masses in Bär et al. (2019) were derived from the measured velocity dispersions of the $\mathrm{Ca} \mathrm{H}, \mathrm{K}$ and $\mathrm{Mg}$ I stellar absorption features, and the employing the relation $\log \left(\mathrm{M}_{\mathrm{BH}} / \mathrm{M}_{\odot}\right)=4.38 \times \log \left(\sigma_{*} / 200 \mathrm{~km} \mathrm{~s}^{-1}\right)+8.49$ found in Kormendy \& Ho (2013).
} 
Table 5. Spectral parameters for LEDA 259433 and LEDA 259580. Model employed is const*phabs*phabs*po.

\begin{tabular}{|c|c|c|c|c|c|c|c|}
\hline Source & $\begin{array}{l}\mathbf{N}_{\mathbf{H}}^{\text {gal. }} \\
\mathbf{c m}^{-2}\end{array}$ & $\begin{array}{c}\mathrm{N}_{\mathrm{H}} \\
\mathbf{c m}^{-2}\end{array}$ & Gamma & $\begin{array}{c}\text { F }_{2-10}^{\mathrm{XRT}} \\
\operatorname{erg} \mathrm{cm}^{-2} \mathrm{~s}^{-1}\end{array}$ & 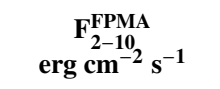 & $\begin{array}{c}\mathrm{F}_{20-100}^{\mathrm{FPMA}} \\
\operatorname{erg} \mathrm{cm}^{-2} \mathrm{~s}^{-1}\end{array}$ & $\chi^{2}$ (d.o.f.) \\
\hline LEDA & $0.076 \times 10^{22}$ (fixed) & $\left(9.43_{-1.54}^{+2.05}\right) \times 10^{22}$ & $1.56 \pm 0.09$ & $\left(2.32_{-0.53}^{+0.72}\right) \times 10^{-12}$ & $\left(2.05_{-0.47}^{+0.63}\right) \times 10^{-12}$ & $\left(0.94_{-0.14}^{+0.41}\right.$ & $142.90(155)$ \\
\hline LEDA 259580 & $0.075 \times 10^{22}$ (fixed) & $\left(25.30_{-4.31}^{+4.87}\right) \times 10^{22}$ & $1.48 \pm 0.12$ & $\left(1.15_{-0.41}^{+0.62}\right) \times 10^{-12}$ & $\left(1.77_{-0.62}^{+0.97}\right) \times 10^{-12}$ & $\left(1.57_{-0.55}^{+0.86}\right) \times 10^{-11}$ & $171.03(149)$ \\
\hline
\end{tabular}

that the IR source is also present in the Wide-field Infrared Survey Explorer (WISE, Wright et al. 2010) catalogue, with magnitudes $\mathrm{w}_{1}=10.966 \pm 0.026, \mathrm{w}_{2}=10.451 \pm 0.024, \mathrm{w}_{3}>10.232$ and $\mathrm{w}_{4}>$ 7.896. The WISE colours, $\mathrm{w}_{1}-\mathrm{w}_{2}=0.515 \pm 0.035$ and $\mathrm{w}_{2}-\mathrm{w}_{3}<$ 0.219 , suggest that Swift $\mathbf{J} 2037.2+4151$ is a stellar object, as inferred by the diagram proposed in Figure 12 of Wright et al. (2010). This supports the previous indication by Landi et al. (2011a) that Swift $\mathrm{J} 2037.2+4151$ is likely a Galactic source, either an X-ray binary or a CV.

The optical/NIR counterpart also appears in the Pan-STARRS catalogue (Chambers et al. 2016) with magnitudes $g>26.3, r>22.4$, $i>20.0, z=21.13 \pm 0.09$ and $y=19.51 \pm 0.13$.

We moreover performed deep optical $R$-band imaging of the field of Swift J2037.2+4151 on Sep. 16, 2014 with the BFOSC instrument (Gualandi 2001) mounted on the 1.5-m "G.D. Cassini" telescope of the INAF-OAS in Loiano (Italy), equipped with a $1300 \times 1340$ pixels EEV CCD with a detector scale of 0.58 pixel $^{-1}$. The observation started at 22:14 UT; three 20-min exposures were collected under an average seeing of 1'.8. After standard debiasing and flat-fielding reduction, the images were stacked together; the photometric analysis was carried out using simple aperture photometry and the field was calibrated using USNO-A2.0 stars located close to the XRT position of Swift J2037.2+4151. No source was detected within the soft X-ray error circle of the object down to a $3 \sigma$ magnitude limit $\mathrm{R}>22.5$. This limit is consistent with that of the Pan-STARRS catalogue at comparable wavebands.

\subsection{Nature and distance of Swift J2037.2+4151}

Assuming from all of the above that Swift J2037.2+4151 is a Galactic object and an X-ray binary, we can infer its nature and distance by placing into context the known multiwavelength information on it.

First, our optical upper limit and the 2MASS near-infrared (NIR) photometry of the source (Skrutskie et al. 2006; see also Landi et al. 2011a) indicates a very red counterpart for this object, which may at least partially be justified by the large absorption along the line of sight detected in the X-rays, as well as by the Galactic reddening $\left(A_{V} \sim 16.6\right.$ mag according to Schlafly \& Finkbeiner 2011; this figure, by the way, fully explains the non-detection of the source in the optical range). However, when we considered the intrinsic NIR colours of stars of different spectral types and luminosity classes as tabulated in Ducati et al. (2001), we immediately found that no combination of reddening, spectral type and luminosity class allows an early-type star as the mass donor in this system: thus, a high-mass $\mathrm{X}$-ray binary nature for this object can be ruled out.

Rather, this reddening implies that the object has intrinsic NIR colours consistent with those of an early M giant according to Ducati et al. (2001): assuming thus that the object has a M2 III star as the NIR counterpart, and that its $V$-band absolute magnitude is -0.6 (Lang 1992), we infer that the distance to the source is $d \approx 10 \mathrm{kpc}$, again using the optical-NIR intrinsic colours of stars tabulated in
Ducati et al. (2001) which imply NIR absolute magnitudes $M_{J}=$ $-3.6, M_{H}=-4.4$ and $M_{K}=-4.5$. This distance would place the source within or just beyond the Cygnus arm of the Galaxy according to the map in e.g. Bodaghee et al. (2012). Late-type dwarf or supergiant interpretations, albeit having similar intrinsic NIR colours, would return distance estimates of $\approx 100$ and $\approx 10^{5}$ pc, respectively: both are untenable either due to the large observed absorption, incompatible with a source relatively close to Earth (in the first case), or because of a position which is too deep into the Galactic halo (in the second). The spectral classification proposed here for the counterpart of Swift $\mathbf{J} 2037.2+4151$ may also be tested via the $Q$ parameter diagnostic (Comerón \& Pasquali 2005; see also Negueruela \& Schurch 2007 and Reig \& Milonaki 2016). Following these authors, one can use the 2MASS NIR photometry Skrutskie et al. (2006) to determine the reddening-free parameter $Q=(J-H)-1.7 \times\left(H-K_{s}\right)$ which, together with the NIR $K_{s}$ magnitude, allows the construction of a diagram in which early-type and late-type stars occupy different loci: while the latter are mostly concentrated around values of $Q$ $=0.4-0.5$ (which correspond to spectral types $\mathrm{K}$ to $\mathrm{M}$ ), early-type objects typically have $Q \sim 0$. In the present case, $Q=0.45 \pm 0.11$, which places the source right in the range of late-type stars.

This spectral classification is also supported by the WISE and Pan-STARRS data: indeed, using the dereddening coefficients of Wang \& Chen (2019), the intrinsic 2MASS NIR magnitudes and the w1-w2 wise colour are compatible with those of a red giant according to Li et al. (2016); likewise, the $z-J$ colour of the source, again corrected for Galactic reddening, is similar to that of a K-M late type star (see Covey et al. 2007). This is the most we could extract from the Pan-STARRS and WISE data, given that no information on the $z$, $y$, w1 and w2 intrinsic absolute magnitudes for Galactic stars is readily available in the literature to the best of our knowledge; therefore, no further support to our distance estimate for Swift J2037.2+4151 could be derived from these catalogues.

We also note, as an aside, that the Galactic hydrogen column density $\mathrm{N}_{\mathrm{H}}$ along the source line of sight is about three times smaller than the one obtained from the dust reddening using the formula of Predehl \& Schmitt (1995): moreover, no stellar type or luminosity class of any kind, as per Ducati et al. (2001), can be recovered by correcting the observed NIR colours with the extinction amount (or lower) associated with the Galactic $\mathrm{N}_{\mathrm{H}}$. This can therefore be considered just a lower limit on the X-ray absorption towards Swift J2037.2+4151. Indeed, the dust reddening we inferred is more compatible with the $\mathrm{N}_{\mathrm{H}}=3.2 \times 10^{22} \mathrm{~cm}^{-2}$ determined by Landi et al. (2011a) and confirmed by our analysis (see 5.2).

Thus, the distance estimate we infer for Swift J2037.2+4151 implies an X-ray luminosity of $\approx 10^{36} \mathrm{erg} \mathrm{s}^{-1}$ in the $2-10 \mathrm{keV}$ band. This is at least a couple of orders of magnitude larger than that of hard X-ray emitting symbiotic stars (i.e., systems composed by a white dwarf accreting from a red giant star - see e.g. Smith et al. 2007; Mukai et al. 2016; Danehkar et al. 2021). Also, this is a factor $\sim 10^{4}$ larger than the typical X-ray output of a CV (see e.g. De Martino et al. 2020): thus, were this source classified as such, it would 
lie at a distance of $\sim 100 \mathrm{pc}$, which (as already stressed above) is too close to justify the large amount of absorption and reddening observed in X-rays and optical/NIR, respectively. Rather, this X-ray output amount seems to be more typical of Symbiotic X-ray binaries, which, despite the similar name, differ from the above class of objects by the fact that the accretor is more compact, namely a neutron star, or even a black hole (see Masetti et al. 2007 for a sample and the main characteristics thereof). This interpretation of course needs a spectroscopic NIR and/or optical confirmation; nevertheless, we deem it as the most viable one to account for the amount of multiwavelegth information we examined here.

\subsection{X-ray data analysis}

In the soft X-rays, apart from the JEM-X detection, Swift J2037.2+4151 has been observed three times by Swift/XRT, twice in 2006 (in August and December, detected at a significance of $46 \sigma$ and $43 \sigma$, respectively) and once in December 2007 (detected at a significance of $22 \sigma$ ), while no observations by other X-ray observatories are present in the archives. In the following, we reanalysed the available XRT data in order to support the classification of Swift J2037.2+4151 based on the multiwavelength characteristics discussed in 5.1 .

We fitted the three XRT datasets separately, since the source might be variable, not only in flux but also in spectral shape in the soft Xrays as well as in the hard X-rays, as expected for Symbiotic X-ray binaries (e.g. Enoto et al. 2014). We followed the same approach for each observation; we initially used a very simple model consisting in a power-law absorbed by intrinsic $\mathrm{N}_{\mathrm{H}}$. As per the discussion in 5.1, we did not add a Galactic $\mathrm{N}_{\mathrm{H}}$ in these and subsequent fits, but we left the component as a free parameter, in order not to over-estimate the value of the intrinsic column density of the source. The simple power-law model does not represent the data well in any of the observations, since residuals around $6 / 7 \mathrm{keV}$, that can be ascribed to the presence of the iron line complex, are clearly visible in the spectra. Therefore we added a Gaussian component to model the residuals; the component is required at more than $99.9 \%$ confidence level in all three spectra, but we found that some residuals are still visible around $7 \mathrm{keV}$ for Observation 1 and 2 . We then added a second Gaussian component in these observations and found that it is required at more than $99.9 \%$ confidence level for Observation 1 spectrum and at more than $99.8 \%$ confidence level for Observation 2 spectrum. The fit results are reported in the upper panel of Table 6 , and as can be seen by the $\chi^{2}$ they are all acceptable. We found that the column density is compatible with the assumptions made in section 5.1. The first emission line feature is found at around $6.4 / 6.5 \mathrm{keV}$, likely corresponding to the highly ionised FeXXIV and/or FeXXV. The second emission line is instead found at $\sim 7 \mathrm{keV}$ and can be associated with FeXXVI. We point out that the Equivalent Widths (EW) we measured for the Gaussian components are slightly larger than what usually found for X-ray binaries (see e.g Masetti et al. 2007 and Onori et al. 2021). This could be due to the fact that we are likely seeing a blending of several lines, which XRT is unable to resolve; this results in a non-physical value of the EW, but nonetheless in line with what is expected in X-ray binaries. In Figure 8 we show the unfolded spectra for the three XRT observations relative to this model. Despite the fits with the simple power-law are quite good, we also tried a more physical approach to our data by fitting them with a thermal model in the form of a blackbody (see lower panel of Table 6), but despite the fits being quite good, we found a blackbody temperature which is unusually high for Low Mass X-ray Binaries (see e.g. Fiocchi et al. 2007).



Figure 8. Swift J2037.2+4151 XRT unfolded spectra. Black spectrum: observation 1; green spectrum: observation 2; red spectrum: observation 3 . The model employed is an absorbed power-law plus two Gaussian components (one in case of Observation 3).

We also checked whether we could apply a Comptonisation model to fit our data (compTT) or a diskbb model; these models are often used to describe the behaviour of X-ray accreting systems and in particular that of Symbiotic X-ray binaries (see Masetti et al. 2007 and Paizis et al. 2006). However, both models do not fit the data sufficiently well and do not allow to put firm constraints on important parameters such as the plasma/disk temperature and optical depth.

From the spectral analysis reported here, it is quite clear that our data lack the statistical significance that would have allowed us to employ more complex and more physical models. Indeed, as is evident from Table 6, the source shows spectral variability, suggesting different states, which need to be further investigated. Spectra of higher quality, taken over different time periods, are therefore essential to better characterise the spectral behaviour of this source. Swift J2037.2+4151 also exhibits flux variability in the $2-10 \mathrm{keV}$ band (see Table 6); the source rises steadily from Observation 1 through to Observation 3. This is an expected behaviour in X-ray binaries, which are known to display variability both on short and long timescales (see e.g. Corbet et al. 2008 and Masetti et al. 2007).

Since the high energy data (INTEGRAL/IBIS and Swift/BAT) are averaged over very long time periods (years), we do not attempt to fit a broad-band spectrum, since variability could be an issue. We therefore analysed data from INTEGRAL/IBIS and Swift/BAT in a similar way as done for IGR J12134-6015, fitting the $20-100 \mathrm{keV}$ spectra together, using a simple power-law (leaving the photon index and the normalisation untied for the two spectra), multiplied by the cflux component, so to have an estimate on the fluxes and their errors. As can be seen from Table 7, the two average spectra are consistent within errors, both in spectral shape and flux.

\section{SUMMARY}

In this paper we have investigated the true nature of three high energy sources. Taking advantage of the multiwavelength data at our disposal, in particular from the NIR to the X-rays, we were able to make robust hypotheses on the classification of the sources we have analysed. 
Table 6. Spectral parameters for Swift J2037.2+4151.

phabs $^{*}(\text { po+ga+ga })^{\star}$

\begin{tabular}{|c|c|c|c|c|c|c|c|c|c|c|}
\hline Obs. & $\begin{array}{c}\mathrm{N}_{\mathrm{H}} \\
\mathrm{cm}^{-2}\end{array}$ & $\Gamma$ & $\begin{array}{l}E_{\text {line }} \\
\text { keV }\end{array}$ & $\begin{array}{c}\sigma \\
\mathbf{e V}\end{array}$ & $\begin{array}{l}\text { EW } \\
\text { eV }\end{array}$ & $\begin{array}{l}E_{\text {line }} \\
\text { keV }\end{array}$ & $\begin{array}{c}\sigma \\
\mathbf{e V}\end{array}$ & $\begin{array}{c}\text { EW } \\
\text { eV }\end{array}$ & $\begin{array}{c}F_{2-10} \\
\operatorname{erg~} \mathrm{cm}^{-2} s^{-1}\end{array}$ & $\chi^{2}$ (d.o.f.) \\
\hline $\begin{array}{l}1 \\
2 \\
3\end{array}$ & $\begin{array}{l}\left(3.99_{-0.53}^{+0.61}\right) \times 10^{22} \\
\left(3.30_{-0.52}^{+0.63}\right) \times 10^{22} \\
\left(3.89_{-0.81}^{+0.98}\right) \times 10^{22}\end{array}$ & $\begin{array}{l}1.07_{-0.21}^{+0.23} \\
0.27_{-0.19}^{+0.20} \\
0.45_{-0.24}^{+0.26}\end{array}$ & $\begin{array}{l}6.41_{-0.10}^{+0.10} \\
6.51_{-0.22}^{+0.13} \\
6.49_{-0.16}^{+0.17}\end{array}$ & $\begin{array}{l}0.10 \text { (fixed) } \\
0.10 \text { (fixed) } \\
0.10 \text { (fixed) }\end{array}$ & $\begin{array}{l}394_{-98}^{+153} \\
288_{-125}^{+139} \\
235_{-174}^{+175}\end{array}$ & $\begin{array}{c}6.94_{-0.06}^{+0.06} \\
7.01_{-0.12}^{+0.10} \\
-\end{array}$ & $\begin{array}{c}0.10 \text { (fixed) } \\
0.10 \text { (fixed) } \\
-\end{array}$ & $\begin{array}{c}737_{-202}^{+201} \\
282_{-158}^{+150} \\
-\end{array}$ & $\begin{array}{l}\left(6.48_{-2.02}^{+2.56}\right) \times 10^{-11} \\
\left(8.73_{-2.20}^{+3.28}\right) \times 10^{-11} \\
\left(1.14_{-0.39}^{+0.65}\right) \times 10^{-10}\end{array}$ & $\begin{array}{l}87.37(90) \\
88.16(80) \\
45.74(57)\end{array}$ \\
\hline \multicolumn{11}{|c|}{ phabs $^{*}(b b+g a+g a)^{\star}$} \\
\hline Obs. & $\underset{\mathrm{Nm}_{\mathrm{H}}^{-2}}{\mathrm{~cm}^{-2}}$ & $\begin{array}{c}\mathrm{kT} \\
\mathrm{keV}\end{array}$ & $\begin{array}{l}E_{\text {line }} \\
\text { keV }\end{array}$ & $\begin{array}{c}\sigma \\
\mathrm{eV}\end{array}$ & $\begin{array}{c}\text { EW } \\
\text { eV }\end{array}$ & $\begin{array}{l}\mathrm{E}_{\text {line }} \\
\text { keV }\end{array}$ & $\begin{array}{c}\sigma \\
\mathbf{e V}\end{array}$ & $\begin{array}{c}\text { EW } \\
\text { eV }\end{array}$ & $\begin{array}{c}F_{2-10} \\
\operatorname{erg~} \mathrm{cm}^{-2} s^{-1}\end{array}$ & $\chi^{2}$ (d.o.f.) \\
\hline $\begin{array}{l}1 \\
2 \\
3\end{array}$ & $\begin{array}{l}\left(2.17_{-0.33}^{+0.38}\right) \times 10^{22} \\
\left(2.22_{-0.35}^{+0.42}\right) \times 10^{22} \\
\left(2.27_{-0.54}^{+0.62}\right) \times 10^{22}\end{array}$ & $\begin{array}{l}1.87_{-0.18}^{+0.20} \\
2.89_{-0.34}^{+0.43} \\
2.75_{-0.36}^{+0.47}\end{array}$ & $\begin{array}{c}6.43 \pm 0.10 \\
6.53_{-0.22}^{+0.12} \\
6.50_{-0.28}^{+0.15}\end{array}$ & $\begin{array}{l}0.10 \text { (fixed) } \\
0.10 \text { (fixed) } \\
0.10 \text { (fixed) }\end{array}$ & $\begin{array}{l}392_{-151}^{+156} \\
272_{-125}^{+135} \\
128_{-112}^{+120}\end{array}$ & $\begin{array}{c}6.94 \pm 0.06 \\
7.02_{-0.11}^{+0.10} \\
-\end{array}$ & $\begin{array}{c}0.10 \text { (fixed) } \\
0.10 \text { (fixed) } \\
-\end{array}$ & $\begin{array}{c}803 \pm 215 \\
280_{-158}^{+150} \\
-\end{array}$ & $\begin{array}{l}\left(5.97_{-0.60}^{+0.76}\right) \times 10^{-11} \\
\left(8.27_{-1.56}^{+2.35}\right) \times 10^{-11} \\
\left(1.11_{-0.23}^{+0.39}\right) \times 10^{-10}\end{array}$ & $\begin{array}{l}98.47(90) \\
85.85(80) \\
46.55(57)\end{array}$ \\
\hline
\end{tabular}

Notes: ${ }^{\star}$ : in the case of Observation 3 data we added only one Gaussian component

Table 7. High energy spectral parameters for Swift J2037.1+4151. Model employed is cflux*po.

\begin{tabular}{ccc}
\hline Instr. & $\Gamma$ & $\begin{array}{c}\mathbf{F}_{\mathbf{2 0}-\mathbf{1 0 0}} \\
\mathbf{e r g ~ c m}^{-\mathbf{2}} \mathbf{s}^{\mathbf{- 1}}\end{array}$ \\
\hline IBIS & $4.76_{-1.26}^{+1.81}$ & $\left(4.71_{-1.28}^{+1.39}\right) \times 10^{-12}$ \\
BAT & $5.71_{-0.31}^{+0.34}$ & $\left(3.94_{-0.23}^{+0.24}\right) \times 10^{-12}$ \\
\hline
\end{tabular}

By employing Gaia measurements, together with optical data obtained at the ESO-VLT telescope, we were able to determine that the proposed classification for IGR J12134-6015 reported in the 157month BAT catalogue is not correct. The source is, in fact, a Galactic object, in particular a Cataclysmic Variable. This is also supported by the X-ray spectra, obtained by the XRT telescope on board the Neil Gehrels Swift Observatory, which can be described by a thermal model typical of CVs; source flux variability is found, as expected for this class of objects.

As far as IGR J16058-7253 is concerned, we were able to assess, thanks to NuSTAR observations, that the hard X-ray emission detected by both INTEGRAL/IBIS and Swift/BAT is not coming from just one of the two counterparts, but rather the high energy detections are the contribution of both AGN. This implies that IGR J16058-7253, or rather one of its counterparts LEDA 259433, cannot be considered as an ultra-luminous AGN, as suggested by Bär et al. (2019). We were able to estimate spectral parameters for both AGN and, using the black hole mass considered by Bär et al. (2019) for LEDA 259433, we also calculated its bolometric and Eddington luminosities, which are well in agreement with the expected values for type $2 \mathrm{AGN}$, confirming our hypothesis that this source does not belong to the class of ultra-luminous active galaxies.

Lastly, multiband analysis of Swift J2037.2+4151 conducted in the optical and X-ray bands, strongly points to this source being part of the rare and peculiar class of Symbiotic X-ray binaries. From the re-analysis of the Swift/XRT data we were able to give a general characterisation of the spectral properties of this source; from our analysis we found that the source is variable both in flux and spectral shape and we also found that Swift J2037.2+4151 exhibits composite features, likely a blending of several emission lines, around the iron line complex. This supports the assumption that Swift J2037.2+4151 is likely a Symbiotic X-ray binary; however more optical, NIR and good quality X-ray data are needed to further support this hypothesis.

\section{ACKNOWLEDGEMENTS}

We thank Ivan Bruni and Silvia Galleti of the Loiano Observatory staff for the assistance during the service mode optical observations.

We thank Gianni Catanzaro for useful hints on finding XShooter data and Gisella Clementini for her support in using the Gaia archive and data.

This research has made use of the 2MASS survey catalogues.

This publication makes use of data products from the Widefield Infrared Survey Explorer, which is a joint project of the University of California, Los Angeles, and the Jet Propulsion Laboratory/California Institute of Technology, funded by the National Aeronautics and Space Administration.

MM acknowledges financial support from ASI/INAF agreement n.2019-35.HH.0

NM acknowledges the ASI financial/programmatic support via the ASI-INAF agreement n.2017-14-H.0 and the 'INAF Mainstream' project on the same subject.

We also thank the anonymous referee for his/her useful comments.

\section{DATA AVAILABILITY}

The data underlying this work are available in the article. Data not specifically appearing in the article like the some X-ray images and hard X-ray spectra will be shared on reasonable request by the corresponding author.

\section{REFERENCES}

Abril J., Schmidtobreick L., Ederoclite A., López-Sanjuan C., 2020, MNRAS, 492, L40

Arnaud K. A., 1996, in G. H. Jacoby \& J. Barnes ed., Astronomical Society of the Pacific Conference Series Vol. 101, Astronomical Data Analysis Software and Systems V. p. 17

Babusiaux C., et al., 2018, A\&A, 616, A10

Bailer-Jones C. A. L., Rybizki J., Fouesneau M., Demleitner M., Andrae R., 2021, AJ, 161, 147 
Bär R. E., et al., 2019, MNRAS, 489, 3073

Barthelmy S. D., et al., 2005, Space Sci. Rev., 120, 143

Baumgartner W. H., Tueller J., Markwardt C., Skinner G., 2010, in AAS/High Energy Astrophysics Division \#11. p. 13.05

Baumgartner W. H., Tueller J., Markwardt C. B., Skinner G. K., Barthelmy S., Mushotzky R. F., Evans P. A., Gehrels N., 2013, ApJS, 207, 19

Bernardini F., de Martino D., Falanga M., Mukai K., Matt G., Bonnet-Bidaud J. M., Masetti N., Mouchet M., 2012, A\&A, 542, A22

Bird A. J., et al., 2006, ApJ, 636, 765

Bird A. J., et al., 2007, ApJS, 170, 175

Bird A. J., et al., 2010, ApJS, 186, 1

Bird A. J., et al., 2016, ApJS, 223, 15

Bodaghee A., Tomsick J. A., Rodriguez J., James J. B., 2012, ApJ, 744, 108

Brown A. G. A., et al., 2021, A\&A, 649, A1

Chambers K. C., et al., 2016, arXiv e-prints, p. arXiv: 1612.05560

Comerón F., Pasquali A., 2005, A\&A, 430, 541

Corbet R. H. D., Sokoloski J. L., Mukai K., Markwardt C. B., Tueller J., 2008, ApJ, 675, 1424

Covey K. R., et al., 2007, AJ, 134, 2398

Danehkar A., Karovska M., Drake J. J., Kashyap V. L., 2021, MNRAS, 500, 4801

De Martino D., Bernardini F., Mukai K., Falanga M., Masetti N., 2020, Advances in Space Research, 66, 1209

Ducati J. R., Bevilacqua C. M., Rembold S. B., Ribeiro D., 2001, ApJ, 558, 309

Enoto T., et al., 2014, ApJ, 786, 127

Eyer L., et al., 2019, A\&A, 623, A110

Fiocchi M., Bazzano A., Ubertini P., Zdziarski A. A., 2007, ApJ, 657, 448

Gehrels N., et al., 2004, ApJ, 611, 1005

Gualandi R. \& Merighi R., 2001, Bologna Astronomical Observatory Technical Report, v2.0

Jayasinghe T., et al., 2018, MNRAS, 477, 3145

Jayasinghe T., Stanek K. Z., Kochanek C. S., 2020, in American Astronomical Society Meeting Abstracts \#235. p. 154.03

Karasev D. I., Lutovinov A. A., Revnivtsev M. G., Krivonos R. A., 2012, Astronomy Letters, 38, 629

Kormendy J., Ho L. C., 2013, ARA\&A, 51, 511

Kretschmar P., et al., 2019, New Astron. Rev., 86, 101546

Krivonos R., Tsygankov S., Revnivtsev M., Grebenev S., Churazov E., Sunyaev R., 2010, A\&A, 523, A61

Krivonos R., Tsygankov S., Lutovinov A., Revnivtsev M., Churazov E., Sunyaev R., 2012, A\&A, 545, A27

Krivonos R. A., et al., 2021, New Astron. Rev., 92, 101612

Landi R., Bassani L., Dean A. J., Bird A. J., Fiocchi M., Bazzano A., Nousek J. A., Osborne J. P., 2009, MNRAS, 392, 630

Landi R., Bassani L., Malizia A., Stephen J. B., Bazzano A., Fiocchi M., Bird A. J., 2010, MNRAS, 403, 945

Landi R., Bassani L., Malizia A., Bazzano A., Fiocchi M., Bird A. J., 2011a, in Extreme and Variable High Energy Sky (Extremesky 2011). p. 9

Landi R., Bassani L., Masetti N., Bazzano A., Bird A. J., 2011b, The Astronomer's Telegram, 3271, 1

Landi R., et al., 2017, MNRAS, 470, 1107

Lang K. R., 1992, Astrophysical Data I. Planets and Stars.

Li J., et al., 2016, ApJ, 823, 59

Lusso E., et al., 2012, Monthly Notices of the Royal Astronomical Society, 425,623

Lutovinov A., Suleimanov V., Manuel Luna G. J., Sazonov S., de Martino D., Ducci L., Doroshenko V., Falanga M., 2020, New Astron. Rev., 91, 101547

Malizia A., Sazonov S., Bassani L., Pian E., Beckmann V., Molina M., Mereminskiy I., Belanger G., 2020, New Astron. Rev., 90, 101545

Marconi A., Risaliti G., Gilli R., Hunt L. K., Maiolino R., Salvati M., 2004, MNRAS, 351, 169

Masetti N., et al., 2007, A\&A, 470, 331

Masetti N., et al., 2013, A\&A, 556, A120

Mukai K., et al., 2016, MNRAS, 461, L1

Negueruela I., Schurch M. P. E., 2007, A\&A, 461, 631

Oh K., et al., 2018, ApJS, 235, 4
Onori F., Fiocchi M., Masetti N., Rojas A. F., Bazzano A., Bassani L., Bird A. J., 2021, MNRAS, 503, 472

Paizis A., et al., 2006, A\&A, 459, 187

Paliya V. S., et al., 2019, ApJ, 881, 154

Predehl P., Schmitt J. H. M. M., 1995, A\&A, 500, 459

Reig P., Milonaki F., 2016, A\&A, 594, A45

Sazonov S., et al., 2020, New Astron. Rev., 88, 101536

Schlafly E. F., Finkbeiner D. P., 2011, ApJ, 737, 103

Skrutskie M. F., et al., 2006, AJ, 131, 1163

Smith R. K., et al., 2007, PASJ, 59, 141

Tueller J., et al., 2006, The Astronomer's Telegram, 853, 1

Ubertini P., et al., 2003, A\&A, 411, L131

Vernet J., et al., 2011, A\&A, 536, A105

Wang S., Chen X., 2019, ApJ, 877, 116

Warner B., 1995, Cataclysmic variable stars. Vol. 28

Westergaard N. J., Budtz-Jorgensen C., Chenevez J., Lund N., Brandt S., Oxborrow C. A., 2006, The Astronomer's Telegram, 967, 1

Winkler C., et al., 2003, A\&A, 411, L1

Wright E. L., et al., 2010, AJ, 140, 1868 